\title{
PROBING DENSITY FLUCTUATIONS IN THE UNIVERSE WITH THE FIRST RADIO SURVEY
}

\author{
C.M.CRESS + FIRST COLLABORATION \\ Astronomy Dept., Columbia Univ., New York, NY10027
}

We compare the angular correlation function measured for FIRST sources (Becker et al., Cress et al.) with COBE-normalized CDM-model predictions (Cress \& Kamionkowski). We note that uncertainties in the z-distribution do not affect the predictions dramatically and that the effects of non-linear evolution of the power spectrum are significant for $\theta<\sim 20^{\prime}$. We find the $C F$ at larger angles to be sensitive to clustering of nearby sources. The smaller angle measurements, when combined with results from other surveys (Loan et al., Rengelink et al.) indicate that the bias required for the data to fit CDM models increases as the surveys probe deeper. We also point the reader to Refregier et al. for information on the use of weak lensing of FIRST sources in probing foreground mass.
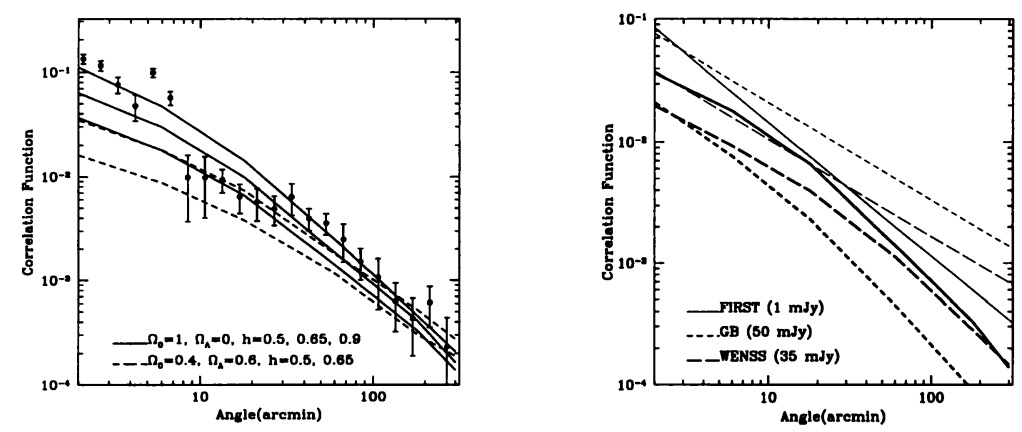

Figure 1. a) Comparison of measured $w(\theta)$ for FIRST ( $1 \mathrm{mJy}$ threshold) with CDM predictions (no bias). b) Power law fits to data (faint lines) and SCDM predictions (no bias) for 3 surveys.

\section{References}

Becker, R. H., White, R. L., \& Helfand, D. J. 1995, ApJ, 450, 559 (BWH)

Cress, C. M., Helfand, D. J., Becker, R. H., Gregg, M. D., \& White, R. L. 1996, ApJ, 473, 7

Cress, C. M. \& Kamionkowski, M., submitted to MNRAS

Refregier, A. et al. 1997, in preparation

Loan A. J., Wall J. V., Lahav O., 1996, MNRAS, 286, 994

Rengelink et al., 1997, in this volume 